\title{
The Essentials of Fitting Hearing Aids to Babies
}

\author{
Marlene P. Bagatto, Au.D., Ph.D. ${ }^{1}$
}

ABSTRACT

Learning Outcomes: As a result of this activity, the participant will be able to: (1) list the four stages of the hearing aid fitting process for infants and (2) describe how fitting hearing aids to infants is different from fitting hearing aids to adults.

For infants with permanent hearing loss, pediatric audiologists strive to initiate intervention by 6 months of age, ${ }^{1}$ and many families choose hearing aid fitting as part of an overall intervention plan. Infants are not simply small adults who need hearing aids; they have different listening needs and present with unique physical and developmental challenges for
${ }^{1}$ National Centre for Audiology, Western University, London, Ontario, Canada.

Address for correspondence and reprint requests: Marlene P. Bagatto, Au.D., Ph.D., National Centre for Audiology, Elborn College, Room 2262J, Western University, London, Ontario, Canada N6G 1H1 (e-mail: bagatto@nca. uwo.ca).
Proceedings of the Widex Audiology Congress; Guest Editor, Robert Sweetow, Ph.D.

Semin Hear 2013;34:19-26. Copyright (C) 2013 by Thieme Medical Publishers, Inc., 333 Seventh Avenue, New York, NY 10001, USA. Tel: +1(212) 584-4662. DOI: http://dx.doi.org/10.1055/s-0032-1333148. ISSN 0734-0451. 
fitting hearing aids compared to adults. For example, an infant's ear canal is constantly growing and changing, which impacts the sound pressure level (SPL) delivered to the ear. Additionally, an infant with hearing loss does not have experience with his or her native language like an adult with acquired hearing loss would. This means that the critical period for language learning must be accessed appropriately so that speech and language development are supported for the infant with hearing loss. Finally, infants are unable to provide verbal feedback about the hearing aid fitting; therefore distinctive strategies are required when adjusting the hearing aid to meet the listening needs of the young preverbal patient.

Thus, the challenging and important work of infant hearing aid fitting requires systematic and pediatric-specific procedures. It is vital, then, to have a comprehensive protocol for fitting hearing aids in infancy to support consistent approaches among clinicians, as well as to effectively reinforce the infant's development of speech and language. Evidence-based protocols and technology exist for the thorough assessment of hearing in infants as well as for accurate and suitable hearing aid fitting in this population. The essential components of fitting hearing aids to babies are the purpose of this article, which is presented in four sections. First, assessment considerations will be discussed in two sections: (1) using electrophysiological threshold estimates for the initial hearing aid fitting and (2) capturing the acoustic properties of infant ear canals. Next, a review of advanced hearing aid technologies and summaries of evidence about their potential use with infants will be provided. Third, a pediatric-friendly strategy for hearing aid verification will be discussed. Finally, an approach to the evaluation of device effectiveness (i.e., validation) will be presented. It is hoped that the clinical relevance of these topics will promote the use of effective strategies for fitting hearing aids to infants.

\section{ASSESSMENT CONSIDERATIONS}

\section{Correcting the Auditory Brainstem Response}

Protocols for infant hearing aid fitting indicate that threshold estimates be obtained for at least two frequencies in each ear. ${ }^{2,3}$ For infants under the age of 6 months, as well as those children who are unable to perform behavioral audiometry reliably (e.g., children with developmental delay), electrophysiologic procedures are conducted. This can include the auditory steadystate response (ASSR), which is calibrated in decibels hearing level ( $\mathrm{dB} H \mathrm{HL})$, or the auditory brainstem response $(\mathrm{ABR})$, which provides an estimate of $\mathrm{dB}$ HL (e.g., referenced in decibels normalized hearing level [dB $\mathrm{nHL}])$. At this stage, the $\mathrm{ABR}$ is a more commonly used procedure to estimate hearing thresholds in infants given that the ASSR requires more investigation for its use in infants with hearing loss. ${ }^{1,4}$ Obtaining threshold estimates using the $\mathrm{ABR}$ provides an appropriate starting point for hearing aid fitting in infants; intervention is not postponed for the collection of behavioral data. An important characteristic of ABR threshold estimates in infants with hearing loss is that they are typically higher (i.e., worse) than what would be obtained through behavioral testing. This is due to various factors such as calibration techniques, level at which the auditory system is assessed, and the duration of the test signal (i.e., long duration pure tones compared with short tone bursts). This discrepancy results in the need for an adjustment to the ABR threshold estimates to better predict behavioral thresholds for the purposes of calculating the prescriptive hearing aid targets. Failing to apply the appropriate correction can result in inaccurate and unsafe hearing aid fittings to infants.

The correction is frequency-specific and is subtracted from the ABR threshold estimate in $\mathrm{nHL}$ to better represent a hearing threshold in dB HL (Fig. 1).

The corrected ABR threshold estimate is referred to in decibels estimated hearing level (dB eHL). The dB eHL value represents an ABR threshold estimate that has been corrected for the purposes of intervention. The correction values are based on the equipment being used to conduct the ABR as well as the collection parameters. A common set of corrections range from 20 at $500 \mathrm{~Hz}$ to 5 at $4000 \mathrm{~Hz}^{5}$ Subtracting these values from the $\mathrm{dB} \mathrm{nHL}$ value serves to better predict the behavioral threshold. This is important because hearing aid prescriptive formula uses behavioral threshold information 


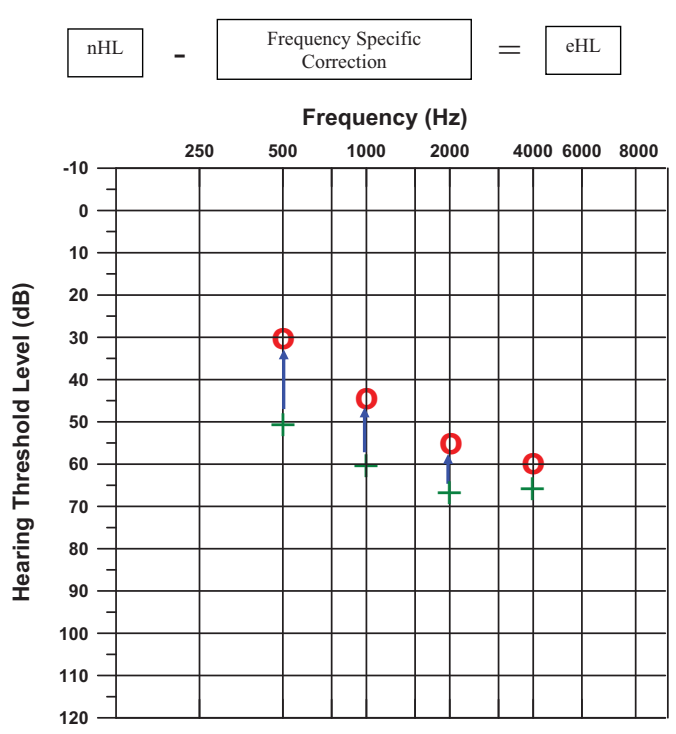

Figure 1 Schematic of how correction factors are applied to auditory brainstem response (ABR) normalized hearing level $(\mathrm{nHL})$ threshold estimation data to estimate behavioral thresholds for the purposes of infant hearing aid fitting. On the audiogram, the plus signs indicate the ABR threshold estimate in $\mathrm{nHL}$, and the circles indicate the corrected value in estimated hearing level. It is important to note that the subtracted corrections improve the initial thresholds estimated by the ABR.

to calculate the prescription. This is because behavioral thresholds are a measure of "true" hearing and not an estimate based on an electrophysiological response (for more on this see Bagatto $^{6}$ ). Properly adjusting the $\mathrm{ABR}$ prior to intervention is a key component in the assessment phase of the hearing aid fitting process for infants.

\section{Capturing Ear Canal Acoustics}

Another assessment consideration when fitting hearing aids to infants is the unique characteristics of the infant ear canal. Growing and changing ear canals impact the SPL delivered to the eardrum and must be accounted for in the hearing aid fitting process. This ensures safe, accurate, and effective levels of sound are being delivered to the infant's tiny ears. It is an important consideration at the assessment stage because the external ear is present and resonates during any audiometric procedure. Therefore, the SPL at the eardrum will vary across indi- viduals for the same hearing level (or estimated hearing level). ${ }^{7}$ Clinically, the way to capture the infant's ear canal acoustics is to measure the real-ear-to-coupler difference (RECD). This is a procedure whereby the SPL in a $2-\mathrm{cm}^{3}$ coupler is subtracted from the SPL measured in the infant's occluded ear canal. ${ }^{8}$ The resulting values are obtained across frequency and referred to as the RECD. The RECD is used in several places in the hearing aid fitting process:

1. To convert audiometry obtained using insert earphones to SPL near the eardrum

2. To convert real-ear gain and output requirements to $2-\mathrm{cm}^{3}$ coupler targets

3. To convert test box measurements of hearing aid output to estimated real-ear measurements

Sample findings from young infants show large variability (i.e., ranging from \pm 12 to \pm 16 depending on frequency) for infants aged 2 to 6 months, which stresses the importance of obtaining the RECD measurement, especially in the first year of life. ${ }^{9}$ Due to the value of measuring the RECD in infants, a useful clinical technique was evaluated to support the clinical application of the measurement, especially in the youngest of patients. ${ }^{10}$ This technique involves connecting the probe tube to a small ear tip or the infant's earmold for insertion into the ear canal simultaneously (Fig. 2).

For an accurate RECD measurement in young infants, the probe tube was noted to be extended beyond the ear tip by $\sim 2$ to $4 \mathrm{~mm}$.

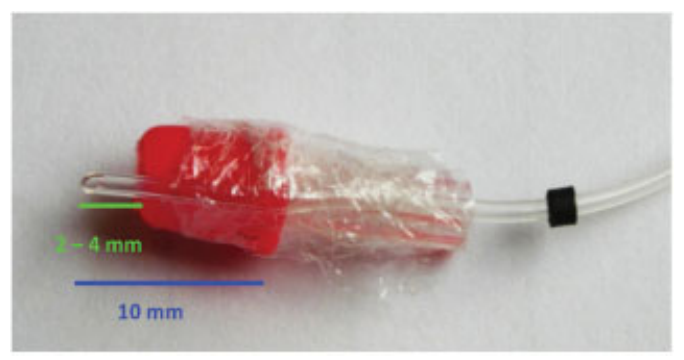

Figure 2 Example of simultaneous probe-tube insertion strategy for use with infants. Insertion depth guidelines are also provided if the clinician chooses to insert the probe-tube independently from the ear tip. 
Alternatively, the probe tube could be inserted into an infant's ear canal $\sim 10 \mathrm{~mm}$ from the opening of the ear canal. This work provides a clinical strategy for measuring the RECD on those patients where it is most critical. The RECD will need to be obtained several times for the same infant given that his or her ear canal is constantly growing. Clinicians should measure the RECD whenever the infant requires a new earmold. This occurs because the infant's ear has grown, which likely results in a change in acoustic properties. Therefore, an RECD is required to account for the changes.

There are, however, practical realities to fitting hearing aids to infants that make measuring the RECD a challenge. Cerumen in the ear canal, middle ear discharge, or an active child may preclude a clinician from obtaining the measurement. Predicted RECD values are available for those instances where the measurement cannot be obtained. ${ }^{9}$ These are provided in applications of the desired sensation level (DSL) method and offer RECD values to the nearest age in months. ${ }^{5,11}$ This is an improvement over previous predicted values that were estimated to the nearest year. Additionally, the current RECD predictions are provided for foam ear tip and earmold coupling. ${ }^{9}$ Limitations of using the predictions are that all children involved in the work had normal middle ear function and high variability was noted in RECD measures associated with children of the same age. ${ }^{9}$ Therefore, whenever possible, predicted values should not replace the more precise RECD measurement when fitting hearing aids to infants.

\section{HEARING AID TECHNOLOGY}

When selecting a hearing aid for an infant with hearing loss, nonelectroacoustic and electroacoustic characteristics are considered. This section will focus on the electroacoustic characteristics; however, it is essential to give mention to the nonelectroacoustic characteristics such as the following:

1. Behind-the-ear (BTE) style

2. Pediatric filtered ear hook

3. Direct audio input
4. Locking mechanism on the battery door and volume control

5. Ability to deactivate advanced features

6. Choice of bright colors

7. Access to loaner devices

These characteristics are mentioned in many pediatric hearing aid fitting protocols and should be considered for every child fitted with a hearing aid. ${ }^{2,3}$

General electroacoustic characteristics of the BTE hearing aid should include the following:

\section{Avoid distortion}

2. Allow frequency/output shaping and flexibility to provide audibility and prevent loudness discomfort

3. Apply wide dynamic range compression for most hearing losses

4. Apply compression output limiting

Pediatric hearing aid fitting protocols support these recommendations based on evidence. ${ }^{2,3}$ The recommendations are achieved by applying an evidence-based prescriptive formula to calculate targets for various levels of speech and maximum power output. Examples of commonly used pediatric prescriptive methods are the DSL method and the National Acoustics Laboratories Nonlinear (NAL-NL2) formula. ${ }^{11,12}$ Through this portion of the selection phase of fitting hearing aids to infants, targets are calculated so that a hearing aid with the appropriate gain characteristics can be chosen. The hearing aid will commonly include advanced technologies for consideration. Unfortunately, the technologies are lacking the research to support their use for infant hearing aid fittings. These technologies include, but are not limited to, feedback management, digital noise reduction, and directional microphones.

Some feedback management strategies work effectively to control feedback without reducing high-frequency output significantly. This is an essential consideration because audibility is a goal for pediatric hearing aid fittings. Applying a feedback manager may be a better option than reducing high-frequency gain manually in the hearing aid fitting software or the 
infant being without a hearing aid while waiting for a new earmold, for example. Consistent hearing aid use may be supported by the application of a feedback-free hearing aid fitting. It should be noted that when feedback management is applied, verification of the output of the hearing aid should be conducted to ensure appropriate audibility across frequencies. ${ }^{13}$ This also is recommended when digital noise reduction (DNR) is applied.

The application of DNR for pediatric hearing aid fittings has been the topic of some recent research. Results indicate that for children ages 5 through 12 years, DNR does not degrade speech recognition abilities and may improve ease of listening in normal hearing children. ${ }^{14,15}$ Similarly for directional microphone use in children, a directional advantage was noted when speech was in front and a disadvantage when speech was behind. ${ }^{16}$ Infants and children do not consistently display appropriate head-turning behaviors, which is vital to ensure optimum directional microphone benefit (i.e., nose to the talker). ${ }^{17,18}$

Limited evidence exists for the impact of advanced technologies on infants, so the technologies should be considered individually, applying solutions with caution and monitoring and verifying their effects. For each technology, it is important to consider the available evidence as well as the individual characteristics of the infant. Additionally, the clinician should consider whether the infant's caregiver can use the technology appropriately and that the infant's access to incidental learning is not compromised as this plays a role in speech and language development. ${ }^{19,20}$ Thus, some pediatric hearing aid fitting protocols have recommended that advanced technologies be considered on a case by case basis. ${ }^{2,3}$ Once the characteristics of the desired hearing aid have been chosen, verification of the output of the hearing aid is required.

\section{HEARING AID VERIFICATION FOR INFANTS}

The main goal of hearing aid verification for infants is to ensure access to the long-term levels of conversational speech while maintaining comfort for loud sounds. ${ }^{5}$ This is done by viewing verification on a sound pressure level (SPL)-ogram for comparison of the hearing aid's output to the infant's thresholds and upper limits (in SPL) as well as for comparison to aided speech targets (Fig. 3).

Viewing hearing aid output on an SPLogram using a real-ear aided response approach allows the clinician to inject meaning into the fitto-targets. Speech-based equipment supports the verification of the hearing aid with meaningful signals for all types of digital hearing aids. Additionally, many hearing aid verification systems support the measurement and application of the RECD so that coupler-based or simulatedreal-ear verification can be conducted. This involves placing the BTE hearing aid on a $2-\mathrm{cm}^{3}$ coupler and applying the RECD values (measured in the assessment stage) so that the output of the hearing aid can be viewed on an SPLogram without the need to have the hearing aid placed on the infant's ear while adjustments are being made. This is critical because infants cannot sit quietly facing a loudspeaker for the duration of actual real-ear verification. Simulated real-ear verification also employs a controlled test-box environment, which is accurate and reliable in children when the RECD is applied. ${ }^{21}$ It is a commonly used procedure for the verification of hearing aids for infants to support early intervention. ${ }^{1}$ Following hearing aid verification, evaluation of the effectiveness of the device is conducted through a systematic and evidencebased validation process.

\section{VALIDATION OF THE HEARING AID FITTING}

The role of validation in the infant hearing aid fitting process is a critical one. Validation allows the clinician to evaluate the outcome of the hearing aid for the infant. Outcomes are important for caregivers and clinicians as well as early hearing detection and intervention (EHDI) programs to track the infant's progress with hearing aids. Until recently, no systematic approach to outcome evaluation has been available for the infant population. The University of Western Ontario Pediatric Audiological Monitoring Protocol (UWO PedAMP) is offered as a guideline for pediatric audiologists to validate hearing aid fittings for children up to 


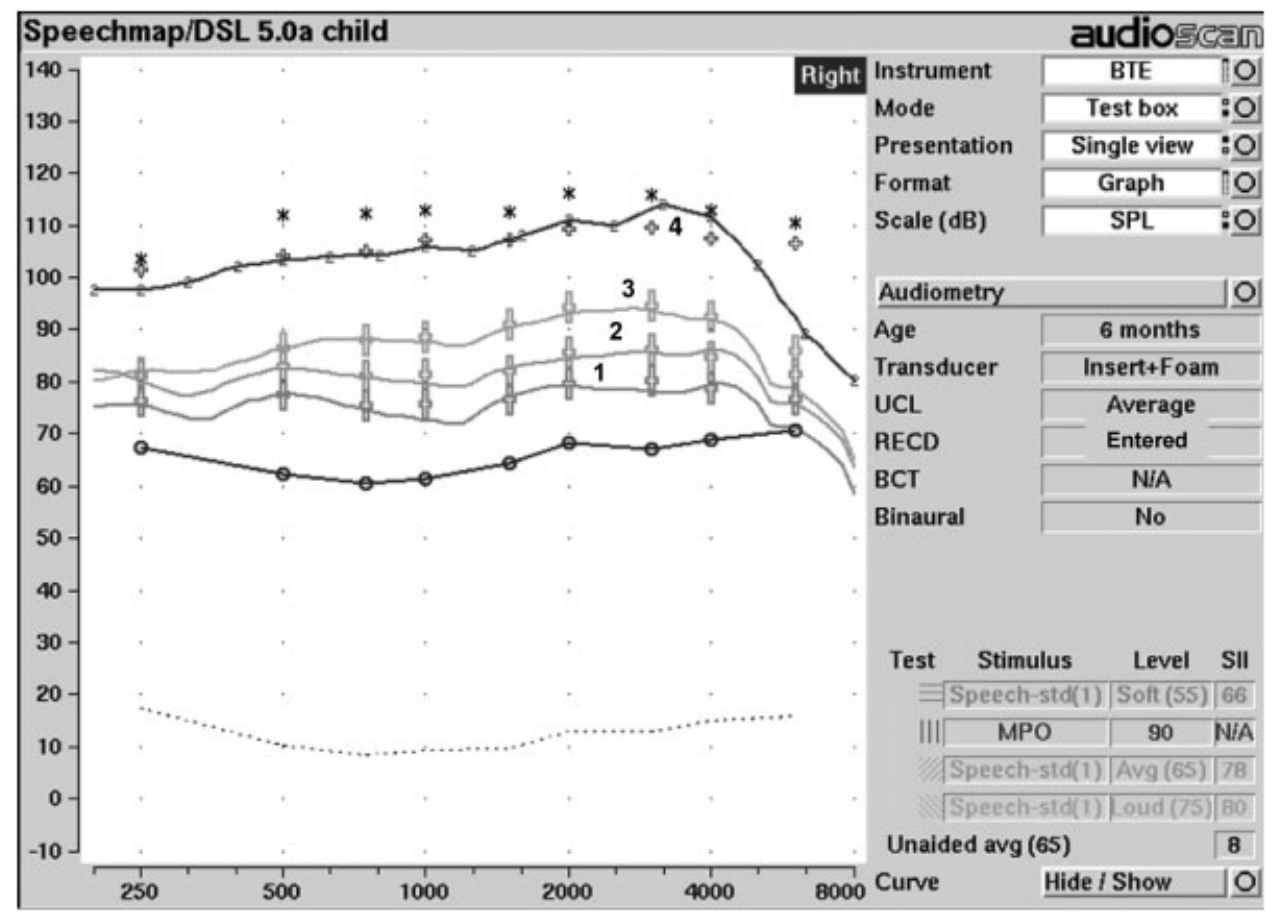

Figure 3 Sound pressure level (SPL)-ogram display of hearing instrument performance in relation to pediatric Desired Sensation Level (DSL) v5.0a targets. The solid lines represent the output of the hearing instrument for soft (1), average (2), and loud (3) speech inputs and maximum power output (4) in relation to the various speech targets (large + ) and maximum power output targets (small + ). Thresholds $(\mathrm{O})$ and upper limits of comfort $\left({ }^{*}\right)$ are also displayed. Abbreviations: BTE, behind the ear; SPL, sound pressure levels; MPO, maximum power output.

the age of 6 years. ${ }^{22}$ The purpose of the UWO PedAMP is to measure the impact of the hearing aid fitting for infants, toddlers, and preschool children. The protocol was developed to be administered and interpreted by the audiologist who fit the hearing aid(s). The tools included within the UWO PedAMP were chosen because they have good statistical properties and are clinically feasible and meaningful. ${ }^{23}$ These characteristics support the clinical uptake of the guideline. ${ }^{24,25}$ The UWO PedAMP includes visual tools to permit rapid scoring of the questionnaires, which helps support its effectiveness and efficiency in clinical practice. In addition, the questionnaires included in the protocol are supported by data from normal hearing as well as hearing-impaired infants who wear hearing aids. ${ }^{22,25-27}$

The UWO PedAMP consists of a tool to assess the clinical process of hearing aid verification (i.e., speech intelligibility index (SII) worksheet) and caregiver-report questionnaires that assess functional outcomes such as auditory development (i.e., LittlEARS) and auditory performance (i.e., Parents' Evaluation of $\mathrm{Au}^{-}$ ral/Oral Performance of Children). Clinical process outcomes are important to consider when interpreting functional outcomes because good quality input from the hearing aid impacts the functional auditory outcomes of the infant. The functional auditory questionnaires were chosen because of their good statistical properties, and they are clinically feasible to administer, score, and interpret for the family. ${ }^{23}$ Details about where to locate each tool within the UWO PedAMP can be found at www.dslio. com. Including a validation protocol in the infant hearing aid fitting completes the process by providing a way to systematically measure the impact of the device for the infant. It also involves caregivers in the process by teaching them to become good observers of their infant's auditory behaviors and fosters a shared language with the audiologist. Outcome evaluation is also important for EHDI programs because it provides a way to measure how the 
program is doing and helps describe patterns that affect infants within the program.

\section{SUMMARY}

Fitting hearing aids to infants with hearing loss is important work for clinicians. Considerations for electrophysiologic testing and unique and changing infant ear canal acoustics must be considered in the assessment stage of the process. Selecting a hearing aid and its appropriate features requires careful consideration of the critical period of speech and language learning as well as the individual caregivers and patients for which the technology is being considered. A crucial stage of the infant hearing aid fitting process involved verifying that the performance of the hearing aid is appropriate for the infant's hearing loss. This will foster good outcomes with the hearing aid that can be systematically evaluated through the use of a newly developed outcome evaluation guideline known as the UWO PedAMP. The significant role of pediatric audiologists is supported by evidence-based and clinically feasible strategies to foster the speech and language skills of hearing-impaired infants.

\section{ACKNOWLEDGMENT}

This work was supported with funding by the Canadian Institutes of Health Research to Marlene Bagatto (200811CGV-204713174463) and the Ontario Research Fund, Early Researcher Award to Susan Scollie, Siemens Hearing Instruments, Canada and The Masonic Foundation of Ontario, Help-2-Hear Project.

\section{REFERENCES}

1. American Academy of Pediatrics, Joint Committee on Infant Hearing. Year 2007 position statement: principles and guidelines for early hearing detection and intervention programs. Pediatrics 2007;120: 898-921

2. American Academy of Audiology (Producer). American Academy of Audiology Pediatric Amplification Guidelines. 2003; Available at: http:// www.audiology.org/resources/documentlibrary/ Documents/pedamp.pdf. Accessed January 7, 2013

3. Bagatto M, Scollie SD, Hyde M, Seewald R. Protocol for the provision of amplification within the Ontario infant hearing program. Int J Audiol 2010;49(Suppl 1):S70-S79
4. Van Maanen A, Stapells DR. Multiple-ASSR thresholds in infants and young children with hearing loss. J Am AcadAudiol 2010;21:535-545

5. Bagatto M, Moodie S, Scollie S, et al. Clinical protocols for hearing instrument fitting in the Desired Sensation Level method. Trends Amplif 2005;9:199-226

6. Bagatto MP. Page ten: baby waves and hearing aids: using ABR to fit hearing aids to infants. The Hearing Journal 2008;6:10,12,14,16

7. Seewald R, Scollie S. Infants are not average adults: implications for audiometric testing. Hear Journal 1999;52:64-72

8. Moodie K, Seewald R, Sinclair S. Procedure for predicting real-ear hearing aid performance in young children. Am J Audiol 1994;3:23-31

9. Bagatto MP, Scollie SD, Seewald RC, Moodie KS, Hoover BM. Real-ear-to-coupler difference predictions as a function of age for two coupling procedures. J Am AcadAudiol 2002;13:407-415

10. Bagatto MP, Seewald RC, Scollie SD, Tharpe AM. Evaluation of a probe-tube insertion technique for measuring the real-ear-to-coupler difference (RECD) in young infants. J Am AcadAudiol 2006;17:573-581

11. Scollie S, Seewald R, Cornelisse L, et al. The Desired Sensation Level multistage input/output algorithm. Trends Amplif 2005;9:159-197

12. Keidser G, Dillon H, Flax M, Ching T, Brewer S. TheNAL-NL2 prescription procedure. Audiol Res 2011;1(1S):e24

13. McCreery R. Pediatric hearing aid verification: Innovative trends. Audiol Online 2008; Available at: http://www.audiologyonline.com/articles/article_detail.asp?article_id=2063. Accessed January 7, 2013

14. Stelmachowicz P, Lewis D, Hoover B, Nishi K, McCreery R, Woods W. Effects of digital noise reduction on speech perception for children with hearing loss. Ear Hear 2010;31:345-355

15. Gustafson SJ, Pittman AL. Sentence perception in listening conditions having similar speech intelligibility indices. Int J Audiol 2011;50:34-40

16. Ricketts T, Galster J, Tharpe AM. Directional benefit in simulated classroom environments. Am J Audiol 2007;16:130-144

17. Ching TY, O'Brien A, Dillon H, et al. Directional effects on infants and young children in real life: implications for amplification. J Speech Lang Hear Res 2009;52:1241-1254

18. Ricketts TA, Galster J. Head angle and elevation in classroom environments: implications for amplification. J Speech Lang Hear Res 2008;51: 516-525

19. Moeller MP, Hoover B, Putman C, et al. Vocalizations of infants with hearing loss compared with infants with normal hearing: Part I-phonetic development. Ear Hear 2007a28:605-627 
20. Moeller MP, Hoover B, Putman C, et al. Vocalizations of infants with hearing loss compared with infants with normal hearing: Part II-transition to words. Ear Hear 2007b28:628-642

21. Seewald RC, Moodie KS, Sinclair ST, Scollie SD. Predictive validity of a procedure for pediatric hearing instrument fitting. Am J Audiol 1999;8: 143-152

22. Bagatto MP, Moodie ST, Malandrino AC, Richert FM, Clench DA, Scollie SD. The University of Western Ontario Pediatric Audiological Monitoring Protocol (UWO PedAMP). Trends Amplif 2011;15:57-76

23. Bagatto MP, Moodie ST, Seewald RC, Bartlett DJ, Scollie SD. A critical review of audiological outcome measures for infants and children. Trends Amplif 2011;15:23-33
24. Moodie ST, Kothari A, Bagatto MP, Seewald R, Miller LT, Scollie SD. Knowledge translation in audiology: promoting the clinical application of best evidence. Trends Amplif 2011;15:5-22

25. Andresen EM. Criteria for assessing the tools of disability outcomes research. Arch Phys Med Rehabil 2000;81(12, Suppl 2):S15-S20

26. Bagatto MP, Brown CL, Moodie ST, Scollie SD. External validation of the LittlEARS Auditory Questionnaire with English-speaking families of Canadian children with normal hearing. Int J PediatrOtorhinolaryngol 2011;75:815-817

27. Bagatto M, Scollie S. Validation of the Parents' Evaluation of Aural/Oral Performance of Children $(\mathrm{PEACH})$ rating scale. J Am AcadAudiol 2013. In press 